\title{
Lord Kelvin's isotropic helicoid
}

Darci Collins, ${ }^{1}$ Rami J. Hamati, ${ }^{1}$ Fabien Candelier, ${ }^{2}$ Kristian Gustavsson, ${ }^{3}$ Bernhard Mehlig ${ }^{\text {a }}{ }^{3}$ and Greg A. Voth ${ }^{\text {b }}$

${ }^{1}$ Department of Physics, Wesleyan University, Middletown, CT 06459, USA

${ }^{2}$ Aix Marseille Univ., CNRS, IUSTI, Marseille, France

${ }^{3}$ Department of Physics, Gothenburg University, 41296 Gothenburg, Sweden

Nearly 150 years ago, Lord Kelvin proposed the isotropic helicoid, a particle with isotropic yet chiral interactions with a fluid, so that translation couples to rotation. An implementation of his design fabricated with a three-dimensional printer is found experimentally to have no detectable translation-rotation coupling, although the particle point-group symmetry allows this coupling. We explain these results by demonstrating that in Stokes flow, the chiral coupling of such isotropic helicoids made out of non-chiral vanes is due only to hydrodynamic interactions between these vanes. Therefore it is small. In summary, Kelvin's predicted isotropic helicoid exists, but only as a weak breaking of a symmetry of non-interacting vanes in Stokes flow.

\footnotetext{
${ }^{a}$ bernhard.mehlig@physics.gu.se

b gvoth@wesleyan.edu
} 


\section{INTRODUCTION}

In his analysis of the forces and torques on a rigid body moving in an incompressible inviscid fluid [1], Lord Kelvin commented on a particular shape, the isotropic helicoid, which experiences the same translational resistance in a homogeneous fluid flow at any orientation, just like a sphere. But unlike a sphere, the particle experiences a torque as it moves through the fluid. To maintain isotropy, this torque must be independent of the particle orientation relative to the flow. This may seem surprising if one takes isotropy to mean continuous rotational symmetry which implies the particle has mirror symmetry and so is non-chiral, which precludes helicity. However Kelvin suggested how to make a helical particle with discrete rotational symmetry and isotropic drag by placing 12 vanes around the great circles of a sphere [1. An implementation following his prescription is shown in Fig. 1)(a).

Since Kelvin's analysis of isotropic helicoids in the inviscid limit, textbooks by Happel \& Brenner [2] and Kim \& Karrila [3] have discussed isotropic helicoids in viscous flows and have concluded that the coupling persists in the low Reynolds number limit. Chiral interactions in turbulent fluids is an area of active research [4, 5. Perturbation theory and numerical simulations have been used to study isotropic helicoids with particle inertia whose translation-rotation coupling causes them to preferentially sample helical regions in viscous flows that are chaotic 6 or turbulent 7 . Quantification of chirality is subtle [8, 9, and translation-rotation coupling of chiral objects is an important test case for proposed measures of chirality [9, 10]. Coupling of translation of chiral particles to rotation and strain is a promising method for hydrodynamic sorting of particles by chirality in viscous flows [11 14.

The elegant theoretical idea of a helical yet isotropic particle has been in the literature for nearly 150 years, cited as an example illustrating the power of symmetry analysis - yet there is no published experimental verification of the conjectured translation-rotation coupling of isotropic helicoids, not in the inviscid limit (Reynolds number Re $\rightarrow \infty$ ), neither in the low-Re limit, nor at any Reynolds number in between. So we 3D-printed different isotropic helicoids and measured their translation-rotation coupling while settling through a quiescent fluid. We tried to achieve as small Re as possible, to avoid confounding effects due to flow separation at large Re, and because the small-Re limit is easiest to analyze theoretically. Our experiments do not show evidence for translation-rotation coupling.

This is surprising, because our particles had the same discrete symmetries as Lord Kelvin's isotropic helicoid. Their point-group symmetry allows translation-rotation coupling, and therefore it is expected to be non-zero in general, provided that there is no other symmetry that forbids this coupling. We explain the experimental null result by showing that there is an additional symmetry that causes the translation-rotation coupling to vanish for helicoids made of non-interacting vanes in the creeping-flow limit. This symmetry is weakly broken by hydrodynamic interactions, which produce a very small coupling, too small measure in our experiments. Our results indicate that the translation-rotation coupling of isotropic helicoids is quite small in general. It remains an open question to engineer isotropic helicoids with maximal coupling.

The remainder of this paper is organized as follows. Section II briefly describes the background, Section [II] gives details about the experiments that resulted in Fig. 1. The theoretical analysis is described in Section IV and Section V] contains conclusions.
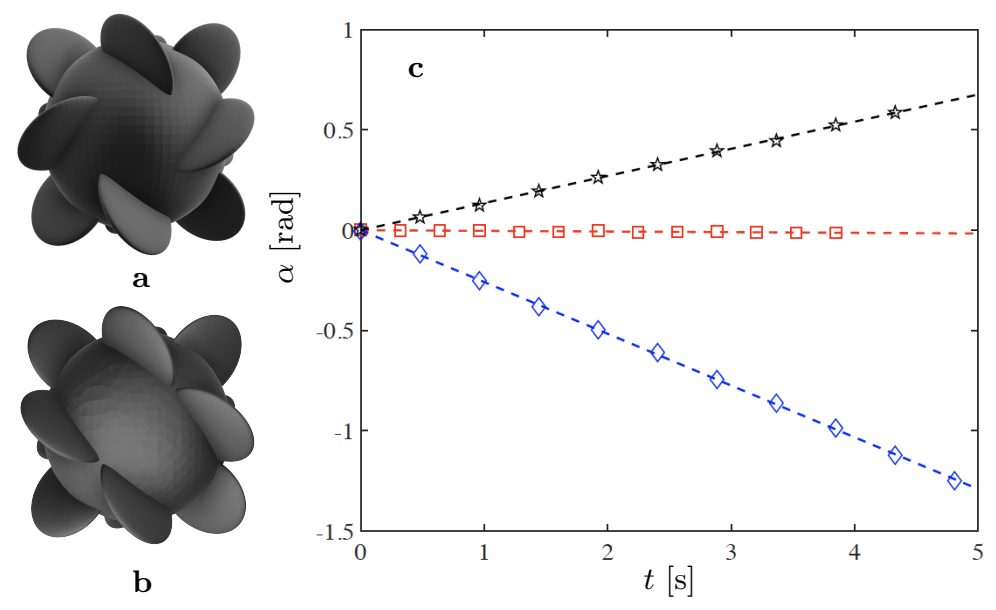

FIG. 1. (a) Model used to 3D-print the left-handed isotropic helicoid. (b) Anisotropic helicoid oriented with the equatorial vanes reflected. (c) Experimental measurements of the particle orientation around its sedimentation axis as a function of time. Isotropic helicoid (red $\square$ with best fit $\omega=\mathrm{d} \alpha / \mathrm{d} t=-0.003 \mathrm{rad} / \mathrm{s}$ ), anisotropic helicoid with initial orientation as in panel $\mathbf{b}$ (blue $\diamond, \omega=-0.258 \mathrm{rad} / \mathrm{s}$ ), and with reflected vanes on a meridian (black $\star, \omega=0.135 \mathrm{rad} / \mathrm{s}$ ). 


\section{BACKGROUND}

\section{A. Force and Torque in the low-Re limit}

In the low Reynolds number limit, the hydrodynamic force $\boldsymbol{f}$ and torque $\boldsymbol{\tau}$ on an arbitrarily shaped particle in a uniform velocity gradient depend linearly on the slip velocity, the angular slip velocity, and upon the local strain rate [2, 3]:

$$
\left[\begin{array}{l}
\boldsymbol{f} \\
\boldsymbol{\tau}
\end{array}\right]=\mu\left[\begin{array}{ccc}
\mathbb{A} & \mathbb{B}^{\top} & \mathbb{G} \\
\mathbb{B} & \mathbb{C} & \mathbb{H}
\end{array}\right]\left[\begin{array}{c}
\boldsymbol{u}^{\infty}-\boldsymbol{v} \\
\boldsymbol{\Omega}^{\infty}-\boldsymbol{\omega} \\
\mathbb{S}^{\infty}
\end{array}\right] .
$$

Here $\mu$ is the dynamic viscosity of the fluid, $\boldsymbol{v}$ and $\boldsymbol{\omega}$ are the velocity and the angular velocity of the particle, $\boldsymbol{u}^{\infty}$ and $\Omega^{\infty}$ are the undisturbed fluid velocity and half the undisturbed fluid vorticity, and $\mathbb{S}^{\infty}$ is the strain-rate tensor, the symmetric part of the matrix of velocity gradients of the undisturbed fluid. Moreover, $\mathbb{A}$ is the drag tensor, $\mathbb{B}$ is the translation-rotation coupling tensor, $\mathbb{C}$ is the rotational drag tensor, and $\mathbb{G}$ and $\mathbb{H}$ are third-rank tensors that couple force and torque to the strain rate. They produce effects such as Jeffery orbits [15, 16], rectification of rotations of chiral dipoles [5], and separation of particles according to chirality [11 14. Here we consider a particle settling steadily in a quiescent fluid, so that $\boldsymbol{u}^{\infty}=0, \boldsymbol{\Omega}^{\infty}=0$, and $\mathbb{S}^{\infty}=0$. In the context of Eq. (1), the question is whether or not Lord Kelvin's particle has non-zero $\mathbb{B}$.

\section{B. Symmetries}

Fig. 2 illustrates the point-group symmetries of different isotropic helicoids. Kelvin's isotropic helicoid places oblique vanes on the 12 edges of an octahedron and has chiral octahedral symmetry, point group O [Fig. 2(a)]. We also fabricated an isotropic helicoid with six four-armed propellers on the faces of a cube (Section III and Appendix D). This particle looks like Fig. 2(b), it also has chiral octahedral symmetry. Panels (c) and (d) illustrate isotropic helicoids with chiral tetrahedral symmetry, point-group T. Either point group, $\mathrm{O}$ or $\mathrm{T}$, constrains $\mathbb{A}$ and $\mathbb{C}$ to be proportional to the unit matrix. Since the groups $\mathrm{O}$ and $\mathrm{T}$ contain rotations only, and no mirror symmetries [18, 19], the tensor $\mathbb{B}$ is constrained in the same way as $\mathbb{A}$ and $\mathbb{C}$. Therefore it must be proportional to the unit matrix. We conclude that the point-group symmetry of these particles ensures isotropy and allows chiral coupling. More detailed calculations arriving at the same conclusion are found in Refs. 2, 3. For convenience, we give the details in Appendix A, for an isotropic helicoid with chiral tetrahedral symmetry. We note that particles with full tetrahedral or octahedral symmetry with reflections constrain the translation-rotation coupling to zero, $\mathbb{B}=0$.

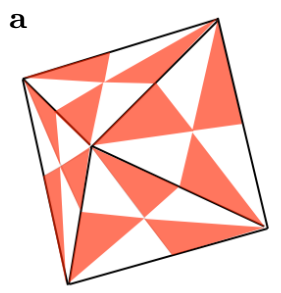

b
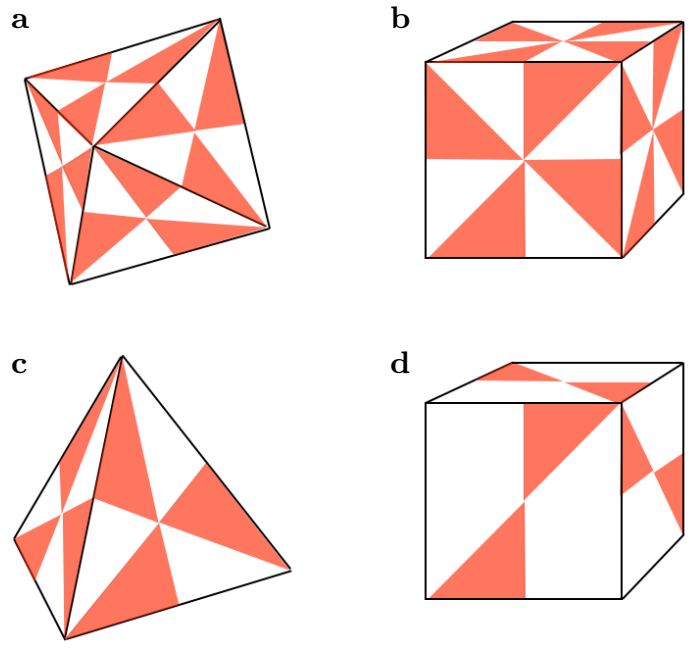

d

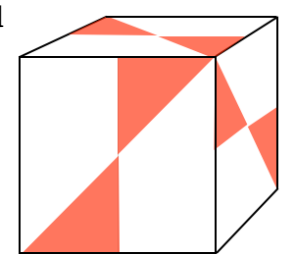

FIG. 2. Illustration of the point-group symmetries of different isotropic helicoids. (a,b) Chiral octahedral symmetry O, four $\mathrm{C}_{3}$ rotation axes, three $\mathrm{C}_{4}$ axes, and six $\mathrm{C}_{2}$ axes. (c,d) Chiral tetrahedral symmetry $\mathrm{T}$, four $\mathrm{C}_{3}$ rotation axes, and three $\mathrm{C}_{2}$ axes (the cubes were drawn after Table 7.2 in Ref. [17]). 


\section{EXPERIMENTS}

We fabricated Lord Kelvin's isotropic helicoid [Fig. 11(a)] using a Form 2 stereolithography 3D printer. For vanes, we used spheroidal disks with diameter $8.7 \mathrm{~mm}$ and aspect ratio 0.2 projecting from the sphere as shown in Fig. 11(a). As proposed by Lord Kelvin, the centers of the disks are equally spaced around the three great circles of a solid sphere with $45^{\circ}$ inclination angle in the direction to create a left-handed particle (using the convention in Ref. [6]) with chiral octahedral symmetry [Fig. 2(a)]. The diameter of the sphere is $d=17.4 \mathrm{~mm}$ and the 3D printed particle has density $\varrho_{\mathrm{p}}=1.16 \mathrm{~g} / \mathrm{cm}^{3}$. When dropped in silicon oil with kinematic viscosity $\nu=5 \mathrm{~cm}^{2} / \mathrm{s}$ and density $\varrho_{\mathrm{f}}=0.98 \mathrm{~g} / \mathrm{cm}^{3}$ in a tank of dimensions $21 \mathrm{~cm}$ wide, $10.5 \mathrm{~cm}$ thick and $30 \mathrm{~cm}$ tall, the particle settles at velocity $v_{s}=4.74 \mathrm{~cm} / \mathrm{s}$. This corresponds to Reynolds number $\operatorname{Re}=d v_{s} / \nu=1.65$.

Figure 1(c) shows the orientation defined as the angle of the particle around the sedimentation axis as it settles approximately $20 \mathrm{~cm}$ through the chamber. Here positive $\alpha$ is the rotation direction favored by a left-handed propeller which is clockwise when viewed from above. The red squares show essentially no change in orientation for the isotropic helicoid with a best fit angular velocity of $\omega=-0.003 \mathrm{rad} / \mathrm{s}$. For comparison, we also printed the anisotropic helicoid shown in Fig. 1(b) with the same dimensions but with the four vanes along one of the great circles reflected to form a right-handed propeller. Separate experiments were performed with the particle oriented along the principal axes of $\mathbb{B}$. When the right-handed great circle is on the equator so that its normal points in the direction of gravity, the particle rotation is shown in blue diamonds. When the right-handed great circle is on a meridian, particle orientation is shown in black triangles. In both cases the sedimentation velocity is within $1 \%$ of that of the isotropic helicoid. Interestingly, the right-handed rotation rate of the anisotropic helicoid is almost exactly twice the two orthogonal left-handed rotation rates, so for randomly oriented particles the total particle helicity would be nearly zero. Experimentally we find that the rotation rate of the isotropic helicoid is near zero, only $1.1 \%$ of the maximum rotation rate of the anisotropic helicoid. The small measured vertical component of the isotropic helicoid rotation rate is twice the random uncertainty in the measurement from video frames, but we observe similar small rotation rates in the other components which are not allowed for homogeneous bodies at low Reynolds numbers and note that density inhomogeneities in the 3D printing are a likely cause. We conclude it is a null detection with approximately $1 \%$ uncertainty.

We tried other versions of isotropic helicoids, but none of them exhibited translation-rotation coupling within measurement error. Four helices extending along tetrahedral angles showed very little rotation but suffered from fabrication imperfections. An isotropic helicoid of six model-airplane propellers in a cubic configuration (details in Appendix D was used in some exploratory experiments on rotation-translation coupling in the high Reynoldsnumber regime. This particle also has chiral octahedral symmetry [Fig. 2(b)]. Its four-bladed propellers (Hobbyking hy 10X8.25) formed a cube with sides $18 \mathrm{~cm}$ which when dropped in air over a distance of $5.5 \mathrm{~m}$ reached a Reynolds number of more than $10^{5}$, but no significant reproducible rotation was observed in several different drop orientations. A single propeller dropped with the blades in the horizontal plane rotated at $46 \mathrm{rad} / \mathrm{s}$ after falling the the same distance in 2.5 seconds. This high Reynolds case where Eq. (1) does not apply is more complicated since there can be separation and symmetry breaking instabilities which can produce tumbling trajectories that couple translation and rotation even for non-chiral particles [20]. Therefore we focus on the low Reynolds number limit in the remainder of this paper.

\section{THEORY}

Our experiments showed no measurable translation-rotation coupling, yet symmetry analysis allows this coupling, as described in Section II. One might argue that the coupling constant could vanish for Lord Kelvin's particle because the vanes around the equator contribute strongly to the expected helical coupling, yet the remaining eight vanes create a torque in the opposite sense, potentially cancelling the coupling. This opposite torque may be familiar to anyone who has observed a propeller move through fluid perpendicular to its usual motion, rotating with helicity opposite to the one usually considered [9].

This is unlikely, however, because of the general principle that says: if symmetry allows a coupling to be non-zero, then this coupling does not vanish unless there is another, undetected symmetry that constrains the coupling to zero. We now demonstrate that there is in fact such an additional symmetry for isotropic helicoids consisting of vanes that do not interact hydrodynamically with each other. In the low-Re limit, hydrodynamic interactions break this additional symmetry only weakly for the isotropic helicoids we considered. This explains why our particles have very small translation-rotation couplings. 


\section{A. Independent vanes}

An isotropic helicoid made out of non-interacting, non-chiral vanes has zero translation-rotation coupling $\mathbb{B}$. To prove this, start with the translation theorem [2, 3] which allows us to relate the tensor $\mathbb{B}$ for a helicoid made out of $M$ non-interacting vanes to the resistance tensors of its vanes, $\mathbb{A}^{(\mathrm{v})}$ and $\mathbb{B}^{(\mathrm{v})}$ :

$$
\mathbb{B}=\sum_{m=1}^{M}\left[\mathbb{O}_{m} \mathbb{B}^{(\mathrm{v})} \mathbb{O}_{m}^{\top}+\boldsymbol{r}_{m} \wedge\left(\mathbb{O}_{m} \mathbb{A}^{(\mathrm{v})} \mathbb{O}_{m}^{\top}\right)\right]
$$

Here $\mathbb{O}_{m}$ is the rotation matrix that rotates from the eigenframe of the isotropic helicoid to that of vane $m, \boldsymbol{r}_{m}$ is the translation vector from the origin of the particle to the centre of the vane, and the matrix $\boldsymbol{v} \wedge \mathbb{W}$ has elements $\varepsilon_{i j k} v_{j} W_{k \ell}$, summation over repeated indices implied. Now a non-chiral vane has $\mathbb{B}^{(\mathrm{v})}=0$ because of mirror symmetry, and the translational resistance tensor is always symmetric $\left[2,\left[\mathbb{A}^{(\mathrm{v})}\right]^{\top}=\mathbb{A}^{(\mathrm{v})}\right.$. Using the antisymmetry of the vector product in the second term on the right hand side of Equation $(2)$ it follows that $\operatorname{Tr} \mathbb{B}=0[21$. Since $\mathbb{B}$ must be proportional to the unit matrix for an isotropic helicoid, it follows that $\mathbb{B}$ is identically zero. A similar result for the rotational coupling of filaments due to collisional momentum transfer is derived in Ref. [10. A consequence is that any helicoid made from non-interacting non-chiral vanes must have zero mean rotation when averaged over all orientations as observed in the experiments.

\section{B. Hydrodynamic interactions}

However, the vanes in a helicoid have hydrodynamic interactions between them. We now show that these interactions produce non-zero chiral coupling as allowed by symmetry, but that the coupling is quite small because the contribution from independent vanes vanishes. To obtain the leading-order hydrodynamic corrections we determine how a given vane $n$ is affected by the disturbance flow created by the other vanes, assuming that the latter are independent. This corresponds to the first-order terms obtained in a systematic expansion in $b / r$ where $b$ is the vane size, and $r$ is the separation between two neighbouring vanes 3 .

Consider an isotropic helicoid made out of $M$ non-chiral vanes. Each vane $m$ has zero translation-rotation coupling, $\mathbb{B}_{m}^{(\mathrm{v})}=0$, and its drag tensor in the frame of the helicoid is denoted by $\mathbb{A}_{m}^{(\mathrm{v})}=\mathbb{O}_{m} \mathbb{A}^{(\mathrm{v})} \mathbb{O}_{m}^{\top}$, where $\mathbb{O}_{m}$ is the appropriate rotation matrix, as defined above. When a single vane moves in a fluid at rest at position $\boldsymbol{x}_{\mathrm{v}}$ with velocity $\boldsymbol{v}_{\mathrm{v}}$, it produces the disturbance flow

$$
\boldsymbol{u}_{\mathrm{v}}^{\prime}(\boldsymbol{x})=\frac{1}{8 \pi \mu} \mathbb{J}\left(\boldsymbol{x}-\boldsymbol{x}_{\mathrm{v}}\right) \boldsymbol{f}_{\mathrm{v}}+\mathscr{O}\left(\frac{1}{\left|\boldsymbol{x}-\boldsymbol{x}_{\mathrm{v}}\right|^{2}}\right)
$$

at position $\boldsymbol{x}$, with $\boldsymbol{f}_{\mathrm{v}}=\mathbb{A}^{(\mathrm{v})} \boldsymbol{v}_{\mathrm{v}}$. Here $(1 / 8 \pi \mu) \mathbb{J}$ is the Green-tensor of the Stokes equation, Equation 4 in Appendix $\mathrm{B}$, and we follow the standard convention, denoting the disturbance flow with a prime.

Each vane moves in the disturbance flow produced by the other vanes. To lowest order in the reflection method, we approximate the force that vane $m$ exerts upon the fluid by

$$
\boldsymbol{f}_{\mathrm{v}, m}=\mathbb{A}_{m}^{(\mathrm{v})}\left(\mathbb{I}-\frac{1}{8 \pi \mu} \sum_{m^{\prime} \neq m}^{M} \mathbb{J}\left(\boldsymbol{x}_{m}-\boldsymbol{x}_{m^{\prime}}\right) \mathbb{A}_{m^{\prime}}^{(\mathrm{v})}\right) \boldsymbol{v}_{\mathrm{v}},
$$

where $\mathbb{I}$ is the identity tensor, $\mathbb{A}_{m}^{(\mathrm{v})} \equiv \mathbb{O}_{m} \mathbb{A}^{(\mathrm{v})} \mathbb{O}_{m}^{\top}$, and $\boldsymbol{x}_{m}$ is the position of vane $m$. Eq. 1 shows that the drag tensor of vane $m$ in the presence of the other vanes is given by $\mathbb{A}_{m}^{(\mathrm{v})}+\delta \mathbb{A}_{m}^{(\mathrm{v})}$ with

$$
\delta \mathbb{A}_{m}^{(\mathrm{v})}=-\frac{1}{8 \pi \mu} \sum_{m^{\prime} \neq m}^{M} \mathbb{A}_{m}^{(\mathrm{v})} \mathbb{J}\left(\boldsymbol{x}_{m}-\boldsymbol{x}_{m^{\prime}}\right) \mathbb{A}_{m^{\prime}}^{(\mathrm{v})}
$$

At leading order in $\left|\boldsymbol{x}_{m}-\boldsymbol{x}_{m^{\prime}}\right|^{-1}$, the drag and translation-rotation coupling tensors of the isotropic helicoid read

$$
\mathbb{A}=\sum_{m=1}^{M} \mathbb{A}_{m}^{(\mathrm{v})}+\delta \mathbb{A}_{m}^{(\mathrm{v})} \text { and } \mathbb{B}=\sum_{m=1}^{M} \boldsymbol{r}_{m} \wedge\left(\mathbb{A}_{m}^{(\mathrm{v})}+\delta \mathbb{A}_{m}^{(\mathrm{v})}\right) \text {. }
$$

The drag tensor $\mathbb{A}$ must be symmetric. Eq. 6 is consistent with this requirement because $\sum_{m=1}^{M} \delta \mathbb{A}_{m}^{(\mathrm{v})}$ is symmetric even though the individual $\delta \mathbb{A}_{m}^{(\mathrm{v})}$ need not be symmetric. As a consequence we find that $\operatorname{Tr} \mathbb{B}=\operatorname{Tr}\left(\sum_{m=1}^{M} \boldsymbol{r}_{m} \wedge \delta \mathbb{A}_{m}^{(\mathrm{v})}\right) \neq$ 

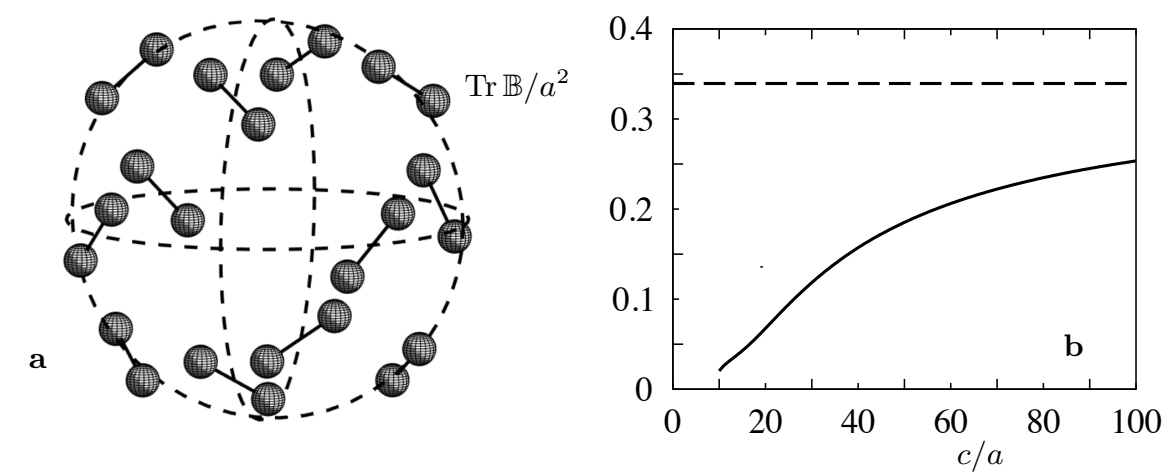

FIG. 3. Theory. (a) Schematic of an isotropic helicoid made out of 24 spheres (radius a) arranged into twelve dumbbells. Each dumbbell (distance $b=5 a$ between centres of spheres) represents a vane of the particle shown in Fig. 1)(a). The dumbbells are assumed to be rigidly connected to each other. Three dashed great circles (radius $c$ ) are guides to the eye. (b) Trace Tr $\mathbb{B}$ of the translation-rotation coupling matrix for the isotropic helicoid from panel a, as a function of particle size $c / a$. Numerical result (solid line), Eqs. (5) and (6) (dashed line).

0 in general. In other words, hydrodynamic interactions between the vanes cause non-zero translation-rotation coupling for an isotropic helicoid.

One might expect that the trace of $\mathbb{B}$ tends to zero as the size of the particle tends to infinity (keeping the vanes unchanged), because hydrodynamic interactions in the low-Re limit are negligible between distant objects. However this argument fails for the translation-rotation coupling. It is true that hydrodynamic interactions decay as $\left|\delta \mathbb{A}_{m}^{(v)}\right| \sim\left|\boldsymbol{r}_{m}\right|^{-1}$, because $\mathbb{J}$ decays in this way. But this decay is cancelled by the magnitude of $\boldsymbol{r}_{m}$ in the vector product in Eq. (6), so that $\operatorname{Tr} \mathbb{B}$ tends to a constant as $\left|\boldsymbol{r}_{m}\right| \rightarrow \infty$. This means that hydrodynamic interactions between the vanes of an isotropic helicoid are not negligible, even if the vanes are very far apart from each other. An explicit calculation for an example is given in Appendix C. We mention that at non-zero Re, the Stokes solution breaks down at large distances, resulting in additional corrections to $\operatorname{Tr} \mathbb{B}$.

\section{Numerical simulations}

To test the theory, Eqs. (5) and (6), we computed higher-order hydrodynamic corrections using the method of Durlofsky et al. 22, a variation of the method of reflections [3. We considered an isotropic helicoid made out of 24 spheres of radius $a$ linked by massless rigid rods [Fig. 3(a)], with the same point-group symmetry as the particle in Fig. 1(a). Each vane is modelled as a dumbbell (length $b=5 a$ ) consisting of two spheres. Each dumbbell is tangential to the surface of an imaginary sphere of radius $c$ (the radius of the isotropic helicoid), and inclined at $45^{\circ}$, just like the vanes in Fig. 1(a). Details of the method are described in Appendix B.

Fig. 3(b) shows our numerical results for the magnitude of the translation-rotation coupling as a function of particle size, $c$. Our numerical results are shown as a solid line, the first-order theory [Eqs. (5) and (6)] as a dashed line. We see that the numerical results approach the theory [Eqs. (5) and [6] at large values of $c$. This is expected, because the contribution of the first reflection must dominate when the vanes are far apart. The convergence is very slow however, the difference between numerical results and lowest-order theory scales as $c^{-1}$ (not shown).

To compare with the experiments, we computed the steady-state angular velocity for the isotropic helicoid in Fig. 3(a). To this end one starts from the expression for the total force $\boldsymbol{F}$ and torque $\boldsymbol{T}$ on a settling particle in a quiescent fluid in the low-Re limit:

$$
\left[\begin{array}{l}
\boldsymbol{F} \\
\boldsymbol{T}
\end{array}\right]=-\mu\left[\begin{array}{cc}
\mathbb{A} & \mathbb{B}^{\top} \\
\mathbb{B} & \mathbb{C}
\end{array}\right]\left[\begin{array}{c}
\boldsymbol{v} \\
\boldsymbol{\omega}
\end{array}\right]+\left(m_{\mathrm{p}}-m_{\mathrm{f}}\right)\left[\begin{array}{l}
\boldsymbol{g} \\
0
\end{array}\right]
$$

The first term represents the hydrodynamic force and torque in the low-Re limit including hydrodynamic interactions, and the second term is due to gravity, with gravitational acceleration $\boldsymbol{g}$ with particle mass $m_{\mathrm{p}}$, and fluid mass $m_{\mathrm{f}}$. To obtain the steady-state angular velocity we must set $\boldsymbol{F}$ and $\boldsymbol{T}$ to zero. The resistance tensor can be inverted using standard formulae [23] for the inversion of block matrices:

$$
\boldsymbol{\omega}=-\mathbb{C}^{-1} \mathbb{B} \boldsymbol{v} \quad \text { with settling velocity } \quad \boldsymbol{v}=\left(m_{\mathrm{p}}-m_{\mathrm{f}}\right) \mu^{-1}\left(\mathbb{A}-\mathbb{B}^{\top} \mathbb{C}^{-1} \mathbb{B}\right)^{-1} \boldsymbol{g} .
$$

We also evaluated the angular velocity of an anisotropic helicoid similar to Fig. 3(a), but with the equatorial dumbbells flipped, as in the experiment. Numerical evaluation of Eq. (8) for these two particles using the method described in 
Appendix $\mathrm{B}$ indicates that the ratio of the angular velocities, isotropic to anisotropic, is non-zero but very small. It is of the order of $10^{-3}$, just below the experimental accuracy.

Numerical evaluation of Eq. (8) also indicates that the magnitude of the translation-rotation coupling and the resulting sense of rotation of the isotropic helicoid depend sensitively on the precise nature of the hydrodynamic interactions. A helicoid with a 25th sphere in the centre of the particle, for example, has the opposite sense of rotation than the particle shown in Fig. 3(a).

Suppose we average the angular velocity of the isotropic helicoid over initial orientations. Our numerical results show that the average angular velocity is not quite zero, because of hydrodynamic interactions. From Eq. (8) we find $\left\langle\omega_{g} / v_{g}\right\rangle=-\operatorname{Tr} \mathbb{C}^{-1} \mathbb{B} \propto \operatorname{Tr} \mathbb{B}$ to leading order, where $v_{g}=\boldsymbol{v} \cdot \hat{\mathbf{g}}, \omega_{g}=\boldsymbol{\omega} \cdot \hat{\mathbf{g}}$, and $\hat{\mathbf{g}}=\boldsymbol{g} / g$. Eq. (6) shows that $\operatorname{Tr} \mathbb{B}$ is non-zero, so that in general $\left\langle\omega_{g} / v_{g}\right\rangle \neq 0$. Our numerical calculations show that similarly $\left\langle\omega_{g}\right\rangle \neq 0$. It could be of interest to explore whether there is an effect analogous to hydrodynamic interactions for the chiral filaments rotating due to momentum transfer from particle collisions [10. A given segment could shield other filament segments from collisions, or give rise to multiple collisions.

\section{CONCLUSIONS}

In conclusion, we measured the dynamics of an isotropic helicoid suggested by Lord Kelvin 150 years ago as it settles in a viscous fluid. Although symmetry analysis indicates that the particle should start to rotate as it settles, we did not detect any translation-rotation coupling in our experiments. This raises the question whether Lord Kelvin's original argument is flawed. Analytic calculation of the rotation-translation coupling tensor for non-interacting, nonchiral vanes shows that the coupling is exactly zero. But taking into account hydrodynamic interactions between vanes reveals non-zero translation-rotation coupling. This coupling is quite weak in general, because it is due to hydrodynamic interactions between the vanes of the isotropic helicoid. The predicted coupling is too weak to be detected in our current generation of experiments.

The possibility of chiral coupling without anisotropy provides an intriguing way to deviate from simple spherical systems, independent from, and in some ways simpler than the much studied case of spheroids. Our discovery of the small size of the chiral coupling helps explain why 150 years after Kelvin first introduced the concept, there are no published measurements of isotropic helicoids. Designing helicoids with optimal chiral coupling provides a challenging focus for future work since it requires designing to control hydrodynamic interaction.

Our results are in keeping with a general rule pertaining to symmetry arguments, seen also in quantum mechanics 24]: If a symmetry allows a matrix element to be non-zero, then it does not vanish unless constrained by some other symmetry. Cases where weak symmetry breaking creates almost zero matrix elements provided deep insights in quantum physics, and we suggest that future work to quantify and optimize isotropic helicoids may also be fruitful.

An important future question is how fluid inertia affects the translation-rotation coupling. Our theory in the low-Re limit explains the experimental findings at small Re. In principle it is possible to include Re-corrections in the theory, but we see no reason why they should change the qualitative conclusions. On the other hand it is interesting to determine whether small-Re corrections decrease or increase the coupling. Our experiments at large Re did not show any evidence for a coupling, but they are far out of the regime of validity of a small-Re expansion.

\section{ACKNOWLEDGMENTS}

We thank S. Östlund, D. Koch, Ji Zhang, and P. Szymczak for discussions. We acknowledge support from NSF grant DMR- 1508575, Army Research Office Grant W911NF-15-1-0205, Vetenskapsrådet [grant number 2017-3865], Formas [grant number 2014-585], and by the grant 'Bottlenecks for particle growth in turbulent aerosols' from the Knut and Alice Wallenberg Foundation, grant number 2014.0048.

[1] Lord Kelvin, "Hydrokinetic solutions and observations," Phil. Mag. 42, 362 (1871).

[2] J. Happel and H. Brenner, Low Reynolds number hydrodynamics: with special applications to particulate media, Vol. 1 (Springer Science \& Business Media, 1983).

[3] S. Kim and S.J. Karrila, Microhydrodynamics: Principles and Selected Applications. 2005 (Dover Publications, 2005).

[4] L. Biferale, S. Musacchio, and F. Toschi, "Split energy-helicity cascades in three-dimensional homogeneous and isotropic turbulence," Journal of Fluid Mechanics 730, 309-327 (2013).

[5] S. Kramel, G. A. Voth, S. Tympel, and F. Toschi, "Preferential Rotation of Chiral Dipoles in Isotropic Turbulence," Phys. Rev. Lett. 117, 154501 (2016). 
[6] K. Gustavsson and L. Biferale, "Preferential sampling of helicity by isotropic helicoids," Phys. Rev. Fluids 1, 054201 (2016).

[7] L. Biferale, K. Gustavsson, and R. Scatamacchia, "Helicoidal particles in turbulent flows with multi-scale helical injection," Journal of Fluid Mechanics 869, 646-673 (2019).

[8] P. W. Fowler, "Quantification of chirality: Attempting the impossible," Symmetry: Culture and Science 16, 321-334 (2005).

[9] E. Efrati and W. T. M. Irvine, "Orientation-dependent handedness and chiral design," Phys. Rev. X 4, 011003 (2014).

[10] G. Dietler, R. Kusner, W. Kusner, E. Rawdon, and P. Szymczak, "Chirality for crooked curves," arxiv:2004.10338 (2020).

[11] Marcos, H. C. Fu, T. R. Powers, and R. Stocker, "Separation of microscale chiral objects by shear flow," Phys. Rev. Lett. 102, $158103(2009)$

[12] R. Eichhorn, "Microfluidic sorting of stereoisomers," Phys. Rev. Lett. 105, 034502 (2010).

[13] S. Meinhardt, J. Smiatek, R. Eichhorn, and F. Schmid, "Separation of chiral particles in micro- or nanofluidic channels," Phys. Rev. Lett. 108, 214504 (2012)

[14] V. Marichez, A. Tassoni, R. P. Cameron, S. M. Barnett, R. Eichhorn, C. Genet, and T. M. Hermans, "Mechanical chiral resolution," Soft Matter 15, 4593-4608 (2019).

[15] G.B. Jeffery, "The motion of ellipsoidal particles immersed in a viscous fluid," Proceedings of the Royal Society of London A: Mathematical, Physical and Engineering Sciences 102, 161-179 (1922).

[16] J. Einarsson, B. M. Mihiretie, A. Laas, S. Ankardal, J. R. Angilella, D. Hanstorp, and B. Mehlig, "Tumbling of asymmetric microrods in a microchannel flow," Phys. Fluids 28, 013302 (2016).

[17] N. W. Ashcroft and N. D. Mermin, Solid State Physics (Holt-Saunders, 1976).

[18] C. W. Curtis and I. Reiner, Representation Theory of Finite Groups and Associative Algebras (Interscience Publishers, 1962).

[19] H. Weyl, Symmetry (Princeton University Press, 1952).

[20] Patricia Ern, Frédéric Risso, David Fabre, and Jacques Magnaudet, "Wake-induced oscillatory paths of bodies freely rising or falling in fluids," Annual Review of Fluid Mechanics 44, 97-121 (2012).

[21] There appears to be a counter example to this theorem in an example in Ref. 3] on page 120 where the translation rotation coupling for a propeller made of unequal size disks has non-zero trace. But it turns out that this is a typographical error with a non-diagonal element printed on the diagonal.

[22] L. Durlofsky, J. F. Brady, and G. Bossis, "Dynamic simulation of hydrodynamically interacting particles," Journal of Fluid Mechanics 180, 21-9 (1987).

[23] T.-T. Lu and S.-H. Shiou, "Inverses of 2 x 2 block matrices," Computers and Mathematics with Applications 43, 119-129 (2002).

[24] E. Merzbacher, Quantum Mechanics (Wiley, 1998).

[25] J. Fries, J. Einarsson, and B. Mehlig, "Angular dynamics of small crystals in viscous flow," Phys. Rev. Fluids 2, 014302 (2017).

[26] wikipedia.org/wiki/Tetrahedron, (2020), [Online; accessed 9-June-2020].

[27] H. Masoud and H. A. Stone, "The reciprocal theorem in fluid dynamics and transport phenomena," Journal of Fluid Mechanics 879, P1 (2019).

[28] F. Candelier and B. Mehlig, "Settling of an asymmetric dumbbell in a quiescent fluid," J. Fluid Mech. 802, 174 (2016).

\section{Appendix A: Tetrahedral symmetry}

We want to deduce the translation-rotation coupling matrix $\mathbb{B}$ for a tetrahedral isotropic helicoid [Fig. 2(c)] from its point-group symmetries. The point-group symmetries of a tetrahedron are summarised in many textbooks, see for example Refs. [18, 19]. The procedure is described in Refs. [2, 3, 25]. One assumes that the particle is at rest, $\boldsymbol{v}=0$ and $\boldsymbol{\omega}=0$. Now one determines how the hydrodynamic force and torque transform under an orthogonal transformatio fn $\mathbb{R}$ corresponding to one of the symmetry operations. If the orientation of the particle relative to the flow remains invariant, the hydrodynamic force becomes simply $\mathbb{R} \boldsymbol{f}$. Since the torque is multiplied by -1 under a reflection, it transforms as $\operatorname{det}[\mathbb{R}] \mathbb{R} \boldsymbol{\tau}$, provided that the orientation of the particle relative to the flow remains unchanged. Inserting this into Eq. (1), and using the orthogonality of $\mathbb{R}$ one finds that the resistance tensors must satisfy the constraints

$$
\mathbb{A}=\mathbb{R} \mathbb{R}^{\top}, \quad \mathbb{B}=\operatorname{det}[\mathbb{R}] \mathbb{R B R}^{\top}, \quad \text { and } \quad \mathbb{C}=\mathbb{R} \mathbb{R}^{\top}
$$

A standard way [26] of parametrizing the corners of the tetrahedral particle shown in Fig. 2(c) is in terms of the median vectors $\boldsymbol{c}_{1}=[1,1,1]^{\top}, \boldsymbol{c}_{2}=[-1,-1,1]^{\top}, \boldsymbol{c}_{3}=[-1,1,-1]^{\top}$, and $\boldsymbol{c}_{4}=[1,-1,-1]^{\top}$ in the Cartesian basis $\hat{\mathbf{x}}$, $\hat{\mathbf{y}}$, and $\hat{\mathbf{z}}$. The particle has chiral tetrahedral point-group symmetry [18, 19]. The symmetry group has 12 elements. Apart from the identity, the group elements are:

i. The $\pi$-rotations around the three bimedians $\frac{1}{2}\left\{\boldsymbol{c}_{1}+\boldsymbol{c}_{4}-\left(\boldsymbol{c}_{2}+\boldsymbol{c}_{3}\right)\right\}, \frac{1}{2}\left\{\boldsymbol{c}_{1}+\boldsymbol{c}_{3}-\left(\boldsymbol{c}_{2}+\boldsymbol{c}_{4}\right)\right\}$, and $\frac{1}{2}\left\{\boldsymbol{c}_{1}+\boldsymbol{c}_{2}-\left(\boldsymbol{c}_{3}+\boldsymbol{c}_{4}\right)\right\}$ (proportional to the three Cartesian coordinate axes). 
ii. Four clockwise rotations around $\boldsymbol{c}_{j}$ by $\frac{2}{3} \pi$.

iii. Four counter-clockwise rotations around $\boldsymbol{c}_{j}$ (by $-\frac{2}{3} \pi$ ).

Since the particle in Fig. 2(c) does not have any mirror symmetries, $\operatorname{det}[\mathbb{R}]=1$ for all symmetry operations in Equation (A1), so that the symmetries constrain $\mathbb{A}, \mathbb{B}$ and $\mathbb{C}$ in the same way. Therefore the translation-rotation coupling $\mathbb{B}$ is constrained to be a multiple of the unit matrix. In general this coupling is expected to be non-zero, as concluded in Refs. [2, 3].

To determine the forms of $\mathbb{A}, \mathbb{B}$, and $\mathbb{C}$ by an explicit calculation, consider first how the $\pi$-rotations (i) around the Cartesian coordinate axes constrain the elements of the resistance tensors $\mathbb{C}$ and $\mathbb{B}$. The corresponding rotation matrices are

$$
\mathbb{R}_{1}=\left[\begin{array}{ccc}
1 & 0 & 0 \\
0 & -1 & 0 \\
0 & 0 & -1
\end{array}\right], \quad \mathbb{R}_{2}=\left[\begin{array}{ccc}
-1 & 0 & 0 \\
0 & 1 & 0 \\
0 & 0 & -1
\end{array}\right], \quad \text { and } \quad \mathbb{R}_{3}=\left[\begin{array}{ccc}
-1 & 0 & 0 \\
0 & -1 & 0 \\
0 & 0 & 1
\end{array}\right]
$$

Inserting these into Equation (A1) we find that the tensors must be diagonal in the body-fixed basis:

$$
\mathbb{A}=\left[\begin{array}{ccc}
A_{11} & 0 & 0 \\
0 & A_{22} & 0 \\
0 & 0 & A_{33}
\end{array}\right], \quad \mathbb{C}=\left[\begin{array}{ccc}
C_{11} & 0 & 0 \\
0 & C_{22} & 0 \\
0 & 0 & C_{33}
\end{array}\right], \quad \text { and } \quad \mathbb{B}=\left[\begin{array}{ccc}
B_{11} & 0 & 0 \\
0 & B_{22} & 0 \\
0 & 0 & B_{33}
\end{array}\right]
$$

Now consider how the symmetries (ii) constrain the tensors. The four rotation matrices (angle $\frac{2}{3} \pi$ ) are

$$
\mathbb{R}_{\boldsymbol{c}_{1}}=\left[\begin{array}{lll}
0 & 0 & 1 \\
1 & 0 & 0 \\
0 & 1 & 0
\end{array}\right], \quad \mathbb{R}_{\boldsymbol{c}_{2}}=\left[\begin{array}{ccc}
0 & 0 & -1 \\
1 & 0 & 0 \\
0 & -1 & 0
\end{array}\right], \quad \mathbb{R}_{\boldsymbol{c}_{3}}=\left[\begin{array}{ccc}
0 & 0 & 1 \\
-1 & 0 & 0 \\
0 & -1 & 0
\end{array}\right], \quad \text { and } \quad \mathbb{R}_{\boldsymbol{C}_{4}}=\left[\begin{array}{ccc}
0 & 0 & -1 \\
-1 & 0 & 0 \\
0 & 1 & 0
\end{array}\right] .
$$

Inserting these into Equation (A1) and using that the tensors $\mathbb{A}, \mathbb{B}$ and $\mathbb{C}$ are diagonal [Equation [A3], we find that any of the symmetries $\mathbb{R}_{\boldsymbol{c}_{j}}$ gives that $A_{11}=A_{22}=A_{33} \equiv A, B_{11}=B_{22}=B_{33} \equiv B$, and $C_{11}=C_{22}=C_{33} \equiv C$. Applying the remaining symmetries does not constrain the elements further.

If the faces of the tetrahedron are non-chiral (they do not have propellers), the particle has a higher symmetry, including in addition 12 mirror symmetries. The corresponding symmetry group is the tetrahedral group. Consider for instance a reflection in the plane spanned by $\boldsymbol{c}_{3}$ and $\boldsymbol{c}_{4}$. Take $\hat{\mathbf{e}}$ to be the unit vector $\hat{\mathbf{e}}=\boldsymbol{c}_{3} \wedge \boldsymbol{c}_{4} /\left|\boldsymbol{c}_{3} \wedge \boldsymbol{c}_{4}\right|$, so that

$$
\hat{\mathbf{e}}=\frac{1}{\sqrt{2}}\left[\begin{array}{l}
1 \\
1 \\
0
\end{array}\right]
$$

Then the reflection matrix is given by $\mathbb{R}_{\hat{\mathbf{e}}}=\mathbb{I}-2 \hat{\mathbf{e}} \hat{\mathbf{e}}^{\top}$,

$$
\mathbb{R}_{\hat{\mathbf{e}}}=\left[\begin{array}{ccc}
0 & -1 & 0 \\
-1 & 0 & 0 \\
0 & 0 & 1
\end{array}\right]
$$

Inserting this matrix into Equation $\left(\mathrm{A} 1\right.$ ) and using that $\mathbb{B}$ and $\mathbb{C}$ are diagonal [Equation $[\mathrm{A} 3$ ] $]$, we find that $B_{33}=0$, but $C_{33}$ is not constrained. The other reflection matrices correspond to other ways of distributing -1 and 1 in distinct rows and columns, different from Equations $(\mathrm{A} 2)$ and $(\mathrm{A} 4)$. These other mirror symmetries constrain $\mathbb{B}=0$, whereas $\mathbb{C}$ is not constrained further, apart from that it must be proportional to the identity. In summary, without propellers we have (in the body-fixed basis)

$$
\mathbb{A}=\left[\begin{array}{ccc}
A & 0 & 0 \\
0 & A & 0 \\
0 & 0 & A
\end{array}\right], \quad \mathbb{C}=\left[\begin{array}{ccc}
C & 0 & 0 \\
0 & C & 0 \\
0 & 0 & C
\end{array}\right], \quad \text { and } \quad \mathbb{B}=0 .
$$

\section{Appendix B: Method for calculating hydrodynamic interactions}

In our numerical simulations, we take into account hydrodynamic interactions using the method of Durlofsky et al. 22. They developed it to determine the evolution of an assembly of free spheres interacting with each other through 
hydrodynamic interactions. In this appendix we briefly summarise their method. We do not include lubrication effects. Consider a single sphere of radius $a_{s}$, at position $\boldsymbol{x}_{s}$, moving with velocity $\boldsymbol{v}_{s}$ in an ambient flow $\boldsymbol{u}^{\infty}$. The disturbance flow produced by this sphere can be determined using the method of singularities, by superimposing a set of singularities built from the Green tensor of the Stokes equations and its spatial derivatives.

Durlofsky et al. approximate the disturbance flow produced by a sphere using a stokeslet, a rotlet, and a stresslet, plus correction terms that take into account the finite size of the sphere. This means that the solution is approximate, but it is accurate enough for our purposes. The disturbance flow due to sphere $s$ reads:

$$
\boldsymbol{u}_{s}^{\prime}(\boldsymbol{x})=\frac{1}{8 \pi \mu}\left[\left(1+\frac{a_{s}}{6} \nabla^{2}\right) \mathbb{J} \boldsymbol{f}_{s}+\mathbb{R} \boldsymbol{\tau}_{s}+\left(1+\frac{a_{s}}{10} \nabla^{2}\right) \mathbb{K}: \mathbb{S}_{s}\right]
$$

where

$$
[\mathbb{J}]_{i j}=\frac{\delta_{i j}}{r}+\frac{r_{i} r_{j}}{r^{3}}, \quad[\mathbb{R}]_{i j}=\frac{1}{2} \varepsilon_{i j k} \frac{r_{k}}{r^{3}}, \quad[\mathbb{K}]_{i j k}=\frac{1}{2}\left(\partial_{k}[\mathbb{J}]_{i j}+\partial_{j}[\mathbb{J}]_{i k}\right), \quad \text { and } \quad r_{i}=\left[\boldsymbol{x}-\boldsymbol{x}_{s}\right]_{i} .
$$

Further, $\boldsymbol{f}_{s}, \boldsymbol{\tau}_{s}$ and $\mathbb{S}_{s}$ are the force, torque, and the stresslet exerted by sphere $s$ upon the fluid. At this stage they are unknown. We mention that $\nabla^{2}[\mathbb{R}]_{i j}$ evaluates to zero, given the expression for the components of $\mathbb{R}$ in Eq. $\mathrm{B} 2$.

Now consider the disturbance flow $\boldsymbol{u}_{s}^{\prime}$ at the centre $\boldsymbol{x}_{s}$ of sphere $s$ that is produced by all other spheres (except sphere $s$ ). This disturbance is obtained by summing Eq. (B1) over $s^{\prime} \neq s$ :

$$
\boldsymbol{u}_{s}^{\prime}=\frac{1}{8 \pi \mu} \sum_{s^{\prime} \neq s}^{N}\left[\left(1+\frac{a_{s^{\prime}}}{6} \nabla^{2}\right) \mathbb{J}\left(\boldsymbol{x}_{s}-\boldsymbol{x}_{s^{\prime}}\right) \boldsymbol{f}_{s^{\prime}}+\mathbb{R}\left(\boldsymbol{x}_{s}-\boldsymbol{x}_{s^{\prime}}\right) \boldsymbol{\tau}_{s^{\prime}}+\left(1+\frac{a_{s^{\prime}}}{10} \nabla^{2}\right) \mathbb{K}\left(\boldsymbol{x}_{s}-\boldsymbol{x}_{s^{\prime}}\right): \mathbb{S}_{s^{\prime}}\right] .
$$

Now force, torque, and stresslet acting upon a sphere moving in a flow can be calculated using the reciprocal theorem 27. Expanding the disturbance velocity $\boldsymbol{u}_{s}^{\prime}$ around the centre of sphere $s$ in order to evaluate the integrals in the reciprocal theorem yields the so-called Faxèn formulae:

$$
\begin{aligned}
\boldsymbol{v}_{s}-\boldsymbol{u}^{\infty} & =\frac{\boldsymbol{f}_{s}}{6 \pi \mu a_{s}}+\left(1+\frac{a_{s}}{6} \nabla^{2}\right) \boldsymbol{u}_{s}^{\prime}, \\
\boldsymbol{\omega}_{s}-\boldsymbol{\Omega}^{\infty} & =\frac{\boldsymbol{\tau}_{s}}{8 \pi \mu a_{s}^{3}}+\frac{1}{2} \boldsymbol{\nabla} \wedge \boldsymbol{u}_{s}^{\prime}, \\
-\mathbb{S}^{\infty} & =-\frac{\mathbb{S}_{s}}{\frac{20}{3} \pi \mu a_{s}^{3}}+\frac{1}{2}\left(1+\frac{a_{s}}{10} \nabla^{2}\right)\left[\boldsymbol{\nabla} \otimes \boldsymbol{u}_{s}^{\prime}+\left(\boldsymbol{\nabla} \otimes \boldsymbol{u}_{s}^{\prime}\right)^{\top}\right],
\end{aligned}
$$

where $\mathbb{S}^{\infty}$ is the symmetric part of the velocity gradient of $\boldsymbol{u}^{\infty}, \boldsymbol{\Omega}^{\infty}=(1 / 2) \boldsymbol{\nabla} \wedge \boldsymbol{u}^{\infty}$, and $\boldsymbol{v}_{s}$ and $\boldsymbol{\omega}_{s}$ are translation and angular velocity of sphere $s$. Eqs. (B4), for $s=1 \ldots N$ constitute a set of linear equations that can be solved for $\boldsymbol{f}_{s}, \boldsymbol{\tau}_{s}$, and $\mathbb{S}_{s}$. Durlofsky et al. [22] explain that the error of the solution scales as $\mathscr{O}\left(\left(\frac{a}{\ell}\right)^{6}\right)$, where $\ell$ is minimal distance between two spheres. This method is quite general. It can be applied to any assembly of free spheres. In our problem the spheres are assumed to be parts of a rigid composite particle. This simply means that all spheres are constrained to move with translation velocity $\boldsymbol{v}_{s}=\boldsymbol{v}+\boldsymbol{\omega} \wedge \boldsymbol{r}_{s}$, and angular velocity $\boldsymbol{\omega}_{s}=\boldsymbol{\omega}$, where $\boldsymbol{v}$ and $\boldsymbol{\omega}$ are the centre-of-mass and angular velocity of the composite particle, and $\boldsymbol{r}_{s}$ parameterizes the location of sphere $s$ w.r.t. the centre of mass of the composite particle. To compute the elements of the force $\boldsymbol{f}$ we impose $\boldsymbol{\omega}=\mathbf{0}$, and $\boldsymbol{v}=[1,0,0]^{\top}, \boldsymbol{v}=[0,1,0]^{\top}, \boldsymbol{v}=[0,0,1]^{\top}$. To compute the torque $\boldsymbol{\tau}$ we impose $\boldsymbol{v}=0$ and $\boldsymbol{\omega}=[1,0,0]^{\top}$, $\boldsymbol{\omega}=[0,1,0]^{\top}, \boldsymbol{\omega}=[0,0,1]^{\top}$. The elements of the coupling tensor are then obtained from Eq. 11. In this way we computed the numerical results shown in Fig. 3

\section{Appendix C: Symmetry breaking due to hydrodynamic interactions}

To illustrate the effect of hydrodynamic interactions, consider a concrete example, a propeller made out of two identical axisymmetric vanes. In the body-fixed basis $(\hat{\mathbf{n}}, \hat{\mathbf{t}}, \hat{\mathbf{b}})$, the drag tensor of a non-chiral vane reads

$$
\left[\mathbb{A}^{(\mathrm{v})}\right]_{i j}=\mu\left[\left(\mathscr{A}_{1}+\mathscr{A}_{2}\right) n_{i} n_{j}+\mathscr{A}_{1}\left(\delta_{i j}-n_{i} n_{j}\right)\right],
$$

where the constants $\mathscr{A}_{1}$ and $\mathscr{A}_{2}$ parametrise the tensor. The centres of the vanes are located at $\boldsymbol{r}_{1}=c \hat{\mathbf{x}}$ and $\boldsymbol{r}_{2}=-c \hat{\mathbf{x}}$ in the lab frame, and they are oriented in such a way that their symmetry axes are orthogonal to $\hat{\mathbf{x}}$. We set $\hat{\mathbf{t}}_{1}=\hat{\mathbf{x}}$ and $\hat{\mathbf{t}}_{2}=-\hat{\mathbf{x}}$, and we rotate each vane around its own vector $\hat{\mathbf{t}}$ by an angle $\phi$, with rotation matrices

$$
\mathbb{O}_{1}=\left[\begin{array}{ccc}
0 & 1 & 0 \\
\cos \phi & 0 & \sin \phi \\
\sin \phi & 0 & -\cos \phi
\end{array}\right] \text { and } \mathbb{O}_{2}=\left[\begin{array}{ccc}
0 & -1 & 0 \\
-\cos \phi & 0 & -\sin \phi \\
\sin \phi & 0 & -\cos \phi
\end{array}\right]
$$




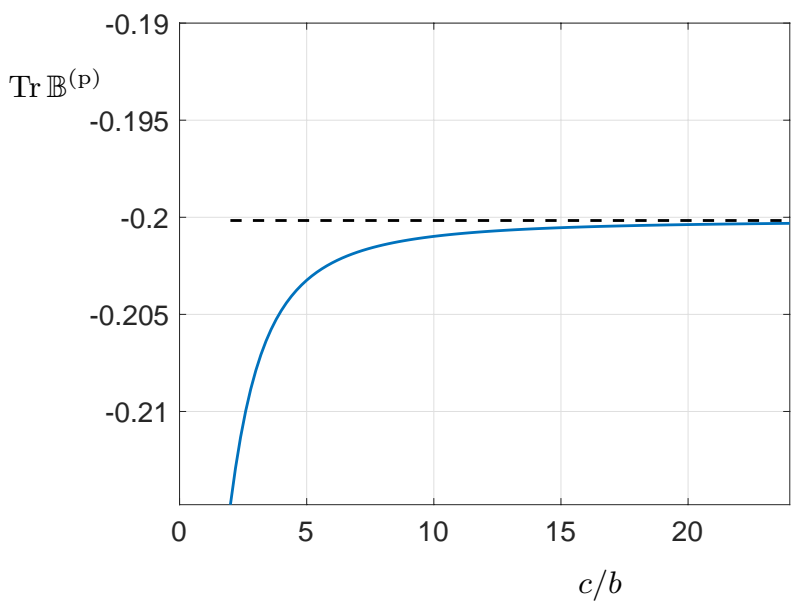

FIG. 4. Numerical result for trace of $\mathbb{B}^{(\mathrm{p})}$ of a two-armed propeller made out of two dumbbells, as a function of the halfdistance between the dumbbells, $c$, normalized by $b$ ( $a$ equals unity), solid line. Dashed-line shows the theoretical large- $c$ limit, Eq. (C5).

Furthermore from Eq. B2

$$
\frac{1}{8 \pi \mu} \mathbb{J}\left(\boldsymbol{r}_{2}-\boldsymbol{r}_{1}\right)=\frac{1}{8 \pi \mu} \mathbb{J}\left(\boldsymbol{r}_{1}-\boldsymbol{r}_{2}\right)=\frac{1}{8 \pi \mu} \frac{1}{2 c}\left[\begin{array}{lll}
2 & 0 & 0 \\
0 & 1 & 0 \\
0 & 0 & 1
\end{array}\right]
$$

Using Eq. (6) we find for the trace of the translation-rotation coupling tensor

$$
\operatorname{Tr} \mathbb{B}^{(\mathrm{p})}=-\frac{1}{8 \pi \mu} \operatorname{Tr}\left(c \hat{\mathbf{x}} \wedge \mathbb{O}_{1} \mathbb{A}^{(\mathrm{v})} \mathbb{O}_{1}^{\top} \mathbb{J O}_{2} \mathbb{A}^{(\mathrm{v})} \mathbb{O}_{2}^{\top}-c \hat{\mathbf{x}} \wedge \mathbb{O}_{2} \mathbb{A}^{(\mathrm{v})} \mathbb{O}_{2}^{\top} \mathbb{J} \mathbb{O}_{1} \mathbb{A}^{(\mathrm{v})} \mathbb{O}_{1}^{\top}\right)
$$

Since the factors of $c$ in this expression cancel out, the trace of $\mathbb{B}^{(p)}$ is independent of $c$ at leading order. This means that the translation-rotation coupling tensor must tend to a constant in the limit of $c \rightarrow \infty$ :

$$
\lim _{c \rightarrow \infty} \operatorname{Tr} \mathbb{B}^{(\mathrm{p})}=\frac{\left(\mathscr{A}_{2}\right)^{2} \sin 4 \phi}{16 \pi}+\mathscr{O}\left(\frac{1}{c}\right) .
$$

In order to check this result, we computed the translation-rotation coupling tensor for a propeller made out of two dumbbells using the method described in Appendix B. The spheres have radius $a$, and the distance $b$ between the spheres that make up the dumbbells is taken to be $5 a$. For this configuration, we find the numerical result $\mathscr{A}_{1}=32.7024 a$ and $\mathscr{A}_{2}=-3.4085 a$ for a single dumbbell. Fig. 4 shows numerical results for Tr $\mathbb{B}(\mathrm{p})$ as a function of $c$ for $\phi=\pi / 3$. We see that the numerical results (solid line) approach the theoretical expecation, Eq. (C5), which evaluates to $\operatorname{Tr} \mathbb{B}^{(\mathrm{p})}=-0.2002 a^{2}$ for $\phi=\pi / 3$. We add the caveat that the above considerations apply to the Stokes limit. If the Reynolds number is non-zero, the Stokes approximation would fail at large distances, requiring a more elaboration calculation 28 .

\section{Appendix D: Exploratory Experiments at larger Reynolds Number}

The four-bladed propeller used in the higher Reynolds-number experiments is shown in Figure 5(a). It is made by Hobbyking and is an hy10x8.25, which indicates 10 inch diameter and 8.25 inch pitch. Six of these propellers on the faces of a cube make an isotropic helicoid with chiral octahedral symmetry as shown in Figure 5 (b). Exploratory experiments dropping this helicoid in air over $5.5 \mathrm{~m}$ reached a Reynolds number of more than $10^{5}$, but showed no reproducible translation-rotation coupling suggesting that the observed suppression of translation-rotation coupling for isotropic helicoids extends to high Reynolds numbers. Further experiments under steady controlled conditions are needed in the high Reynolds number regime. 

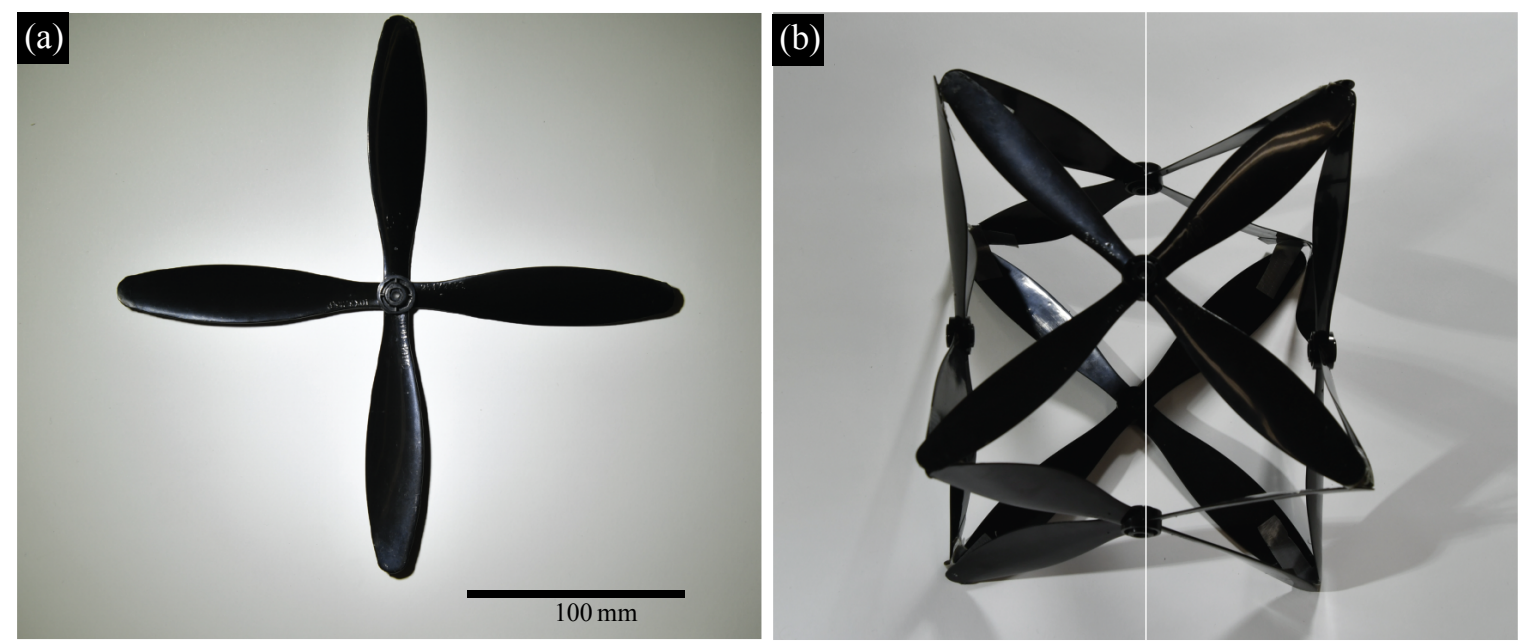

FIG. 5. Higher-Re experiments. (a) single four-bladed propeller. (b) the cubic isotropic helicoid constructed from six of the propellers shown in panel (a). 